\title{
Multi-criteria analysis of ten single family houses regarding environmental impacts
}

\author{
Andrea Moňoková1, and Silvia Vilčeková ${ }^{1, *}$ \\ ${ }^{1}$ Technical University of Košice, Faculty of Civil Engineering, Institute of Environmental \\ Engineering, Vysokoškolská 4, 04200, Košice, Slovakia
}

\begin{abstract}
This study presents a life cycle assessment (LCA) of ten single family houses located in Eastern Slovakia with the aim to compare them in terms of the materials and technologies used. The main goal is to investigate and emphasize the reduction rate of environmental impact resulting from using green materials and technologies. Environmental impacts are determined by using eToolLCD software. Life cycle impact assessment (LCIA) categories of global warming, ozone depletion, acidification, eutrophication and photochemical ozone creation potential, as well as abiotic depletion potential - elements, abiotic depletion potential - fossil fuels, use of renewable primary energy resources, net use of fresh water, components for reuse and materials for recycling are determined within the cradle-to-grave boundary. Assessed family houses are built as a combination of conventional materials such as aerated concrete blocks, expanded polystyrene (EPS), extruded polystyrene (XPS) and roofing mineral wool and natural materials such as wood, cellulose, clay, straw and extensive vegetation roofs. Multi-criteria decision analysis points out that material optimization of building structures as well as the application of green technologies can ensure a considerable reduction of environmental impacts.
\end{abstract}

\section{Introduction}

Buildings and construction activities are playing important role in urbanisation by creating residential and working spaces and contributing to the state economy [1,2]. However, they also have serious negative impact on the natural environment. Construction, operation and demolition of buildings, leads to large amount of noise, dust, water pollution and waste production [3]. Besides that, these buildings represent the largest energy-consuming sector ( $35 \%$ of overall global energy consumption) and they make significant contribution to $\mathrm{CO} 2$ production. International Energy Agency predicts, that energy consumption in building sector will increase by $50 \%$ by 2050 , if no other actions to enhance buildings energy efficiency will be made [4]. Green buildings are an effort to lower the negative impacts on environment and at the same time to increase positive effects of buildings during their life cycle. Concept of green building includes not only "sustainability", but also "high energy performance", which means, that energy efficiency could not be at the costs of decreasing

\footnotetext{
Corresponding author: silvia.vilcekova@tuke.sk
} 
quality of indoor environment nor level of comfort [5,6,7]. However, green buildings still represent only a small portion of the building stock in both developed and developing countries $[8,9]$. Some studies indicate the need to create market mechanism to support the development of green buildings, whereas governmental regulation and grants are necessary only in case of market failure $[7,8,10]$. That is why, to support these buildings in marketoriented economics is essential to convince markets participants that "green could become gold" [11].

Subject of the paper is life cycle assessment of ten family houses from Eastern Slovakia using the LCA method. This analysis is focused on environmental impact categories: global warming potential (GWP), eutrophication potential (EP), acidification potential (AP), photochemical ozone creation potential (POCP), ozone depletion potential (ODP), abiotic depletion potential - elements (ADPE), abiotic depletion potential - fossil fuels (ADPF), net use of fresh water (FW), use of renewable primary energy resource, as raw materials (PERM) use of renewable primary energy resource, as energy source (PERE), components for reuse (CRU) and materials for recycling (MFR).

\section{Methodology}

The methodology framework in this research for environmental impacts benchmarking of ten houses with conventional and green materials has been explained in this methodology section.

\subsection{Definition of goal and scope}

Each of the family houses is built by combination of conventional building materials and natural so-called green materials. Conventional systems and also systems using renewable sources are used for heating and domestic hot water heating (DHW). Combination of materials and energy sources is different in each case. The aim of environmental impact comparison, of assessed family houses is:

- $\quad$ Finding, which phase of family house's life cycle causes the greatest environmental damage.

- Determination, which building materials are contributing to these damages the most.

- Assessment, which environmental problem is the most significant in relation to family house and thus it needs to be prioritized.

- Evaluation whether the financial resources invested in eco-innovations, are going to bring significant improvement in environmental impacts, or more precisely to look for the most appropriate combination of constructional materials and energy resources, to achieve the lowest environmental impacts at acceptable financial costs. This could considerably help not only in decision making process of architects and civil engineers, but also to investors themselves.

- Support the development of technologies and processes of production of new environmentally friendly products or materials.

\subsection{Method}

LCA analysis of selected family houses was performed using eToolLCD software [12], which is working according to norm EN ISO 14044 and EN 15978. Software provides results of evaluation using the CML-IA methodology. It uses third-party background 
processes aggregated as midpoint indicators, stored in software library that are associated with algorithms and user inputs to produce an environmental impact assessment.

Chosen functional unit for each family house is $1 \mathrm{~m} 2$ of the total floor area, which represents base to which the inputs and outputs of modelling the benchmark system relate.

Length of the referential period was set to 60 years, which is according to certification system BREEAM (Building Research Establishment Environmental Assessment Method).

To make it obvious, what will be and what will not be the subject of the inventory in the inventory phase, was necessary to define, which stages of life cycle would be subject of the analysis. In ideal case it should be considered all the stages and processes from extracting the primary resources, including necessary materials, product production stage, operation stage and final disposal. The larger the system boundaries, the more complicated the study is, the more demanding the input data and the processing time. That is why the system boundary was narrowed to phases most probably different for each studied house. Selected phases are described in Table 1.

Table 1. System boundaries based on cradle-to-grave LCA.

\begin{tabular}{|c|c|c|c|}
\hline \multicolumn{2}{|c|}{ Life Cycle Stage } & Detail Description & Code \\
\hline \multirow{5}{*}{ Stage before Use } & \multirow{3}{*}{ Product Stage } & Raw Material Extraction & A1 \\
\hline & & Raw Material Transport & A2 \\
\hline & & Product Manufacturing & A3 \\
\hline & \multirow{2}{*}{ Construction } & Product/ Equipment Transport & A4 \\
\hline & & Construction - Installation & A5 \\
\hline \multirow{5}{*}{\multicolumn{2}{|c|}{ Uses Stage }} & Use & B1 \\
\hline & & Maintenance & B2 \\
\hline & & Refurbishment & B5 \\
\hline & & Integrated Operational Energy & B6 \\
\hline & & Operational Water & B7 \\
\hline \multicolumn{2}{|c|}{ End of Life Stage } & Disposal & $\mathrm{C} 4$ \\
\hline
\end{tabular}

\section{Inventory analysis}

Key step of the inventory is to quantify the amount of raw materials and quantities of emitted substances to environment, which are in relation to functional unit, expressed by referential flow of assessed product. Software eToolLCD uses the available database BRE IMPACT, version 4. To display the resulting data by the amount of environmental impacts for each category, the so-called inventory tables are used. 


\subsection{Required data for inventory analysis}

After defining the goal and scope of the LCA study, the next step was to collect the material and energy data of the investigated buildings for inventory analysis. Following table 2 shows basic information about assessed family houses (FH), used constructional materials and their heating systems.

Table 2. Brief description of materials and energy source of family houses.

\begin{tabular}{|c|c|c|c|c|}
\hline FH & External walls & Roofs & Floors & $\begin{array}{l}\text { Services } \\
\text { equipment }\end{array}$ \\
\hline 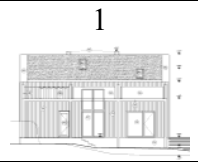 & $\begin{array}{l}\text { clay poured into the } \\
\text { shell, timber cladding, } \\
\text { clay plaster }\end{array}$ & $\begin{array}{l}\text { extensive vegetation, } \\
\text { waterproofing, mineral } \\
\text { wool, rafter framework- } \\
\text { saddle roof }\end{array}$ & $\begin{array}{l}\text { wood, } \\
\text { ceramic } \\
\text { tiles, EPS }\end{array}$ & gas boiler \\
\hline & $\begin{array}{l}\text { wooden framed } \\
\text { structure, straw } \\
\text { insulation, timber } \\
\text { cladding, clay plaster }\end{array}$ & $\begin{array}{l}\text { extensive vegetation, } \\
\text { wooden fibre insulation, } \\
\text { waterproofing, rafter } \\
\text { framework-saddle roof }\end{array}$ & $\begin{array}{l}\text { wood, } \\
\text { ceramic } \\
\text { tiles, EPS }\end{array}$ & gas boiler \\
\hline r sum & $\begin{array}{l}\text { wooden framed } \\
\text { structure, straw } \\
\text { insulation, timber } \\
\text { cladding, clay plaster }\end{array}$ & $\begin{array}{l}\text { extensive vegetation, } \\
\text { mineral wool, } \\
\text { waterproofing, rafter } \\
\text { framework-saddle roof }\end{array}$ & $\begin{array}{l}\text { wood, } \\
\text { ceramic } \\
\text { tiles, EPS }\end{array}$ & $\begin{array}{l}\text { air-to-water } \\
\text { heat pump, } \\
\text { solar } \\
\text { collectors }\end{array}$ \\
\hline & $\begin{array}{l}\text { wooden framed } \\
\text { structure, wooden fibre } \\
\text { insulation, timber } \\
\text { cladding, plasterboard }\end{array}$ & $\begin{array}{l}\text { extensive vegetation, } \\
\text { waterproofing, cellulose, } \\
\text { reinforced concrete }\end{array}$ & $\begin{array}{l}\text { wood, } \\
\text { ceramic } \\
\text { tiles, EPS }\end{array}$ & $\begin{array}{l}\text { air-to-water } \\
\text { heat pump }\end{array}$ \\
\hline & $\begin{array}{l}\text { CLT wood system, sheep } \\
\text { wool, timber cladding }\end{array}$ & $\begin{array}{l}\text { extensive vegetation, } \\
\text { waterproofing, PUR, } \\
\text { CLT wood system }\end{array}$ & $\begin{array}{l}\text { wood, } \\
\text { ceramic } \\
\text { tiles, EPS }\end{array}$ & $\begin{array}{l}\text { air-to-water } \\
\text { heat pump, } \\
\text { solar } \\
\text { collectors }\end{array}$ \\
\hline & $\begin{array}{l}\text { aerated concrete blocks, } \\
\text { wooden fibre slabs, } \\
\text { silicate plaster }\end{array}$ & $\begin{array}{l}\text { concrete tiles, } \\
\text { waterproofing, cellulose, } \\
\text { rafter framework-hip roof }\end{array}$ & $\begin{array}{l}\text { wood, } \\
\text { ceramic } \\
\text { tiles, EPS }\end{array}$ & $\begin{array}{l}\text { air-to-water } \\
\text { heat pump, } \\
\text { solar } \\
\text { collectors }\end{array}$ \\
\hline min & $\begin{array}{l}\text { aerated concrete blocks, } \\
\text { wooden fibre slabs, } \\
\text { silicate plaster }\end{array}$ & $\begin{array}{l}\text { concrete tiles, } \\
\text { waterproofing, cellulose, } \\
\text { rafter framework-hip roof }\end{array}$ & $\begin{array}{l}\text { wood, } \\
\text { ceramic } \\
\text { tiles, EPS }\end{array}$ & gas boiler \\
\hline 8 & $\begin{array}{l}\text { reinforced concrete, } \\
\text { ceramics bloks, mineral } \\
\text { wool, XPS, cilicate } \\
\text { plaster }\end{array}$ & $\begin{array}{l}\text { ceramics tiles, } \\
\text { waterproofing, EPS, } \\
\text { reinforced concrete }\end{array}$ & $\begin{array}{l}\text { wood, } \\
\text { ceramic } \\
\text { tiles, EPS }\end{array}$ & gas boiler \\
\hline$\frac{9}{\square \square}$ & $\begin{array}{l}\text { ceramics bloks, mineral } \\
\text { wool, cilicate plaster }\end{array}$ & $\begin{array}{l}\text { extensive vegetation, } \\
\text { waterproofing, EPS, } \\
\text { reinforced concrete }\end{array}$ & $\begin{array}{l}\text { wood, } \\
\text { ceramic } \\
\text { tiles, EPS }\end{array}$ & $\begin{array}{l}\text { air-to-water } \\
\text { heat pump }\end{array}$ \\
\hline men & $\begin{array}{l}\text { aerated concrete blocks, } \\
\text { EPS, silicate plaster }\end{array}$ & $\begin{array}{l}\text { modified bitumen strip, } \\
\text { waterproofing, EPS, } \\
\text { reinforced concrete }\end{array}$ & $\begin{array}{l}\text { wood, } \\
\text { ceramic } \\
\text { tiles, EPS }\end{array}$ & gas boiler \\
\hline
\end{tabular}

The selected family houses are located in villages near towns of Košice (FH 4 - 10), Bardejov (FH 1) and Gelnica (FH 2, 3) in Eastern Slovakia. 


\section{Life cycle assessment}

Informational overview of environmental impact categories, quantified from whole referential period of analysed $\mathrm{FH}$ is presented in table 3. It was performed by eToolLCD software.

Table 3. Environmental impact categories of $\mathrm{RH}$ quantified for $\mathrm{m} 2$ total floor area.

\begin{tabular}{|c|c|c|c|c|c|c|c|c|c|c|}
\hline FH & 1 & 2 & 3 & 4 & 5 & 6 & 7 & 8 & 0 & 10 \\
\hline Category of Impact & & & & & & & $r$ & 0 & & \\
\hline $\begin{array}{c}\mathbf{G W P} \\
\mathrm{kg} \mathrm{CO} \\
\mathrm{eq}\end{array}$ & 1400 & 330 & 1400 & 1600 & 970 & 1700 & 1400 & 1300 & 1400 & 1900 \\
\hline $\begin{array}{c}\mathbf{A P} \\
\mathrm{kg} \mathrm{SO}_{2 \mathrm{eq}}\end{array}$ & 2,8 & 2,3 & 9,9 & 8,9 & 7,1 & 7,7 & 2,7 & 3,4 & 6,9 & 3,5 \\
\hline $\begin{array}{c}\text { EP } \\
\mathrm{kg} \mathrm{PO}_{4}^{3-}{ }_{\text {eq }}\end{array}$ & 0,91 & 1,2 & 3,4 & 2,0 & 1,9 & 2,5 & 0,7 & 0,92 & 1,7 & 1 \\
\hline $\begin{array}{l}\text { ADPE } \\
\mathrm{kg} \mathrm{Sb} b_{\text {eq }}\end{array}$ & $1,5 \mathrm{E}^{-3}$ & $1,4 \mathrm{E}^{-3}$ & $6,8 \mathrm{E}^{-3}$ & $4,6 \mathrm{E}^{-3}$ & $8,5 \mathrm{E}^{-3}$ & $2,0 \mathrm{E}^{-3}$ & $9,7 \mathrm{E}^{-4}$ & $1,7 \mathrm{E}^{-3}$ & $2,6 \mathrm{E}^{-3}$ & $1,8 \mathrm{E}^{-3}$ \\
\hline $\begin{array}{c}\text { ADPF } \\
\text { MJ }\end{array}$ & 6700 & 5400 & $29,0 \mathrm{E}^{3}$ & $26 \mathrm{E}^{3}$ & $21 \mathrm{E}^{3}$ & $23 \mathrm{E}^{3}$ & 6900 & 8800 & $19,0 \mathrm{E} 3$ & 9700 \\
\hline $\begin{array}{c}\text { ODP } \\
\mathrm{kg} \mathrm{CFC} 11_{\mathrm{eq}}\end{array}$ & $2,4 \mathrm{E}^{-5}$ & $2,1 \mathrm{E}^{-5}$ & $1,1 \mathrm{E}^{-3}$ & $0,8 \mathrm{E}^{-3}$ & $1,2 \mathrm{E}^{-3}$ & $5,6 \mathrm{E}^{-4}$ & $6,4 \mathrm{E}^{-5}$ & $2,7 \mathrm{E}^{-5}$ & $0,4 \mathrm{E}^{-3}$ & $6,4 \mathrm{E}^{-5}$ \\
\hline $\begin{array}{c}\text { POCP } \\
\mathrm{kg} \mathrm{C}_{2} \mathrm{H}_{4 \mathrm{eq}}\end{array}$ & 0,42 & 0,33 & 0,96 & 0,63 & 0,86 & 0,67 & 0,38 & 0,53 & 0,62 & 0,59 \\
\hline $\begin{array}{l}\text { PERM } \\
\text { MJ }\end{array}$ & 0,64 & $2,7 \mathrm{E}^{-3}$ & 0,02 & 0,012 & $7,4 \mathrm{E}^{-3}$ & 0,05 & $5,6 \mathrm{E}^{-3}$ & 0,021 & $9,5 \mathrm{E}^{-3}$ & 0,053 \\
\hline $\begin{array}{l}\text { PERE } \\
\text { MJ }\end{array}$ & 2200 & 4500 & 9100 & 2700 & 2100 & 820 & 2600 & 740 & 780 & 1200 \\
\hline $\begin{array}{c}\mathbf{F W} \\
\mathrm{m}^{3}\end{array}$ & 87 & 760 & 130 & 140 & 110 & 110 & 87 & 40 & 65 & 62 \\
\hline $\begin{array}{c}\text { CRU } \\
\mathrm{kg}\end{array}$ & 38 & 13 & 4,9 & 18 & 60 & 130 & 60 & 15 & 45 & 37 \\
\hline $\begin{array}{c}\text { MFR } \\
\mathrm{kg}\end{array}$ & 1800 & 900 & 1400 & 2100 & 2600 & 4100 & 1800 & 2000 & 1800 & 3200 \\
\hline
\end{tabular}

GWP - global warming potential, AP - acidification potential, EP - eutrophication potential, ADPE - abiotic depletion potential - elements, ADPF - abiotic depletion potential - fossil fuels, ODP - ozone depletion potential,

POCP - photochemical ozone creation potential, PERM - use of renewable primary energy resource, as raw materials, PERE - use of renewable primary energy resource, as energy source, FW - net use of fresh water, CRU - components for reuse, MFR - materials for recycling

FH 2 and 6 (table 4,5) were chosen to present the results of the environmental impact assessment, which were evaluated by the multi-criteria decision analysis as the best (2) and the most inappropriate (6) alternative. There were options of $10 \mathrm{FH}$ in decision making process. These options were evaluated from the best option to the worst option by CDA (Concordance Dis-concordance Analysis) multicriterial decision-making method with socalled cardinal information about criteria. For the calculation, 12 environmental impact categories were selected as criterion (Table 3) to which so-called weighting of criteria according to importance so that the sum is equal to $100 \%$. Individual criteria were divided into maximization or minimization according to their nature.

The cells highlighted in colour in the table show the level of impact of each indicator evaluated.

It is velar from presented tables, that the largest contributors to environmental impacts are phases: product manufacture (A1-A3), operational energy use (B6) and to large extend also renovation phase (B5). In case of FH 2, which is largely made of "green" materials, the impacts on the global warming potential (GWP) are lowest. Comparable to FH 6 it is up to $80 \%$ less. Also, in case of acidification potential (AP) and eutrophication potential (EP) is 
FH 2 better alternative. AP/EP potential values are 70\%/52\% lower than FH 6 . It seems that this difference appears due to the use of different heating sources (FH 2 - gas boiler, RD 6 - air-to-water heat pump).

Out of materials, the biggest contributor to GWP is concrete. It represents $18-29 \%$ of all materials used. A high share on this impact has also thermal insulation made of extruded polystyrene $(2,1-3,6 \%)$ and PP or PVC foil (1,6-4,5\%). Other GWP contributors are aluminium window frames and concrete roof tiles.

Table 4. Environmental impact categories for each phase of life cycle-FH 2 evaluated as the best option.

\begin{tabular}{|c|c|c|c|c|c|c|c|c|c|c|}
\hline \multirow{2}{*}{$\begin{array}{l}\text { Category } \\
\text { of Impact }\end{array}$} & \multicolumn{3}{|c|}{ Stage before Use } & \multicolumn{5}{|c|}{ Use Stage } & \multirow{2}{*}{$\begin{array}{c}\begin{array}{c}\text { End of } \\
\text { Life Stage }\end{array} \\
\text { C4 } \\
\end{array}$} & \multirow{2}{*}{ Total } \\
\hline & $\mathbf{A} 1-\mathbf{A} \mathbf{3}$ & A4 & A5 & B1 & B2 & B5 & B6 & B7 & & \\
\hline $\begin{array}{c}\text { GWP } \\
\mathrm{kg} \mathrm{CO}_{2 \mathrm{eq}}\end{array}$ & -110 & 29 & 11 & 0 & 0.14 & 20 & 360 & 12 & 12 & 330 \\
\hline $\begin{array}{c}\text { AP } \\
\mathrm{kg} \mathrm{SO}_{2 \mathrm{eq}}\end{array}$ & 1.40 & 0.068 & 0.073 & 0 & $6.4 \mathrm{E}^{-4}$ & 0.54 & 0.10 & 0.06 & 0.072 & 2.30 \\
\hline $\begin{array}{c}\text { EP } \\
\mathrm{kg} \mathrm{PO}_{4}^{3-}{ }_{\mathrm{eq}}\end{array}$ & 0.39 & 0.015 & 0.045 & 0 & $1.5 \mathrm{E}^{-4}$ & 0.13 & 0.025 & 0.014 & 0.58 & 1.20 \\
\hline $\begin{array}{l}\text { ADPE } \\
\mathrm{kg} \mathrm{Sb}_{\mathrm{eq}}\end{array}$ & $7.8 \mathrm{E}^{-4}$ & $0.1 \mathrm{E}^{-5}$ & $0.3 \mathrm{E}^{-6}$ & 0 & $0.14 \mathrm{E}^{-7}$ & $0.6 \mathrm{E}^{-3}$ & $0.12 \mathrm{E}^{-5}$ & $2.8 \mathrm{E}^{-6}$ & $3.7 \mathrm{E}^{-8}$ & $1.4 \mathrm{E}^{-3}$ \\
\hline $\begin{array}{c}\text { ADPF } \\
\text { MJ }\end{array}$ & 3400 & 410 & 150 & 0 & 2.20 & 1100 & 180 & 190 & 0.40 & 5400 \\
\hline $\begin{array}{c}\text { ODP } \\
\operatorname{kg~CFC11} 1_{\mathrm{eq}}\end{array}$ & $1.2 \mathrm{E}^{-5}$ & $1.9 \mathrm{E}^{-6}$ & $6.3 \mathrm{E}^{-7}$ & 0 & $0.7 \mathrm{E}^{-8}$ & $0.5 \mathrm{E}^{-5}$ & $0.6 \mathrm{E}^{-6}$ & $6.1 \mathrm{E}^{-7}$ & $7.8 \mathrm{E}^{-9}$ & $2.1 \mathrm{E}^{-5}$ \\
\hline $\begin{array}{c}\text { РОСР } \\
\mathrm{kg} \mathrm{C}_{2} \mathrm{H}_{4 \mathrm{eq}} \\
\end{array}$ & 0.21 & 0.013 & 0.012 & 0 & $3.6 \mathrm{E}^{-5}$ & 0.07 & 0.014 & $3.5 \mathrm{E}^{-3}$ & $5.2 \mathrm{E}^{-3}$ & 0.33 \\
\hline $\begin{array}{c}\text { PERM } \\
\text { MJ }\end{array}$ & $2.1 \mathrm{E}^{-3}$ & $0.4 \mathrm{E}^{-5}$ & $6.5 \mathrm{E}^{-6}$ & 0 & $3.0 \mathrm{E}^{-7}$ & $4.8 \mathrm{E}^{-4}$ & $2.6 \mathrm{E}^{-5}$ & $2.5 \mathrm{E}^{-5}$ & $7.7 \mathrm{E}^{-7}$ & $2.7 \mathrm{E}^{-3}$ \\
\hline $\begin{array}{c}\text { PERE } \\
\text { MJ }\end{array}$ & 4200 & 0.61 & 0.95 & 0 & 0.041 & 200 & 3.50 & 8 & 0.13 & 4500 \\
\hline $\begin{array}{c}\mathbf{F W} \\
\mathrm{m}^{3}\end{array}$ & 360 & 0.046 & 0.08 & 0 & $3.4 \mathrm{E}^{-3}$ & 360 & 0.29 & 38 & $4.4 \mathrm{E}^{-4}$ & 760 \\
\hline $\begin{array}{c}\text { CRU } \\
\mathrm{kg}\end{array}$ & 0 & 0 & 1.2 & 0 & 0 & 0 & 0 & 0 & 12 & 132 \\
\hline $\begin{array}{c}\text { MFR } \\
\mathrm{kg}\end{array}$ & 0 & 0 & 51 & 0 & 0 & 37 & 0 & 0 & 810 & 900 \\
\hline
\end{tabular}

Impact key red: nad $60 \%$, orange: $51-60 \%$, violet: $41-50 \%$, yellow: $31-40 \%$, green: $21-30 \%$, blue: $10-20 \%$ 
Table 5. Environmental impact categories for each phase of life cycle - FH 6 evaluated as the worse option.

\begin{tabular}{|c|c|c|c|c|c|c|c|c|c|c|}
\hline \multirow{2}{*}{$\begin{array}{l}\text { Category } \\
\text { of Impact }\end{array}$} & \multicolumn{3}{|c|}{ Stage before Use } & \multicolumn{5}{|c|}{ Use Stage } & \multirow{2}{*}{\begin{tabular}{|c}
$\begin{array}{c}\text { End of } \\
\text { Life Stage }\end{array}$ \\
C4
\end{tabular}} & \multirow{2}{*}{ Total } \\
\hline & $\mathbf{A} 1-\mathbf{A} 3$ & A4 & A5 & B1 & B2 & B5 & B6 & B7 & & \\
\hline $\begin{array}{c}\text { GWP } \\
\mathrm{kg} \mathrm{CO}_{2 \mathrm{eq}}\end{array}$ & 520 & 59 & 25 & -12 & 0.033 & 92 & 1000 & 24 & 22 & 1700 \\
\hline $\begin{array}{c}\mathbf{A P} \\
\mathrm{kg} \mathrm{SO} \mathrm{SO}_{2 \mathrm{q}}\end{array}$ & 2 & 0.14 & 0.18 & 0 & $1.5 \mathrm{E}^{-4}$ & 0.51 & 4.8 & 0.12 & 0.053 & 7.70 \\
\hline $\begin{array}{c}\text { EP } \\
\mathrm{kg} \mathrm{PO}_{4}^{3-} \text { eq }\end{array}$ & 0.46 & 0.65 & 0.045 & 0 & $3.5 \mathrm{E}^{-6}$ & 0.17 & 1.10 & 0.027 & 0.087 & 2.50 \\
\hline $\begin{array}{l}\text { ADPE } \\
\mathrm{kg} \mathrm{Sb}_{\mathrm{eq}}\end{array}$ & $1.3 \mathrm{E}^{-3}$ & $3.5 \mathrm{E}^{-5}$ & $2.1 \mathrm{E}^{-7}$ & 0 & $3.3 \mathrm{E}^{-9}$ & $6.7 \mathrm{E}^{-4}$ & $1.0 \mathrm{E}^{-4}$ & $5.6 \mathrm{E}^{-6}$ & $2.4 \mathrm{E}^{-8}$ & $2.0 \mathrm{E}^{-3}$ \\
\hline $\begin{array}{c}\text { ADPF } \\
\text { MJ }\end{array}$ & 5000 & 830 & 360 & 0 & 0.2 & 970 & 16000 & 370 & 0.23 & 23000 \\
\hline $\begin{array}{c}\text { ODP } \\
\operatorname{kg~CFC} 11_{\mathrm{eq}}\end{array}$ & $2.3 \mathrm{E}^{-5}$ & $5.5 \mathrm{E}^{-3}$ & $1.6 \mathrm{E}^{-6}$ & 0 & $1.7 \mathrm{E}^{-9}$ & $3.1 \mathrm{E}^{-5}$ & $5.2 \mathrm{E}^{-5}$ & $1.2 \mathrm{E}^{-6}$ & $6.1 \mathrm{E}^{-9}$ & $5.6 \mathrm{E}^{-3}$ \\
\hline $\begin{array}{c}\text { РОCP } \\
\mathrm{kg} \mathrm{C}_{2} \mathrm{H}_{4 \mathrm{eq}}\end{array}$ & 0.28 & 0.64 & 0.034 & 0 & $8.7 \mathrm{E}^{-6}$ & 0.053 & 0.27 & $6.8 \mathrm{E}^{-3}$ & $2.3 \mathrm{E}^{-3}$ & 1.30 \\
\hline $\begin{array}{c}\text { PERM } \\
\text { MJ }\end{array}$ & 0.047 & 0.62 & $4.3 \mathrm{E}^{-6}$ & 0 & $7.3 \mathrm{E}^{-8}$ & $8.9 \mathrm{E}^{-3}$ & $2.3 \mathrm{E}^{-3}$ & $4.9 \mathrm{E}^{-5}$ & $4.8 \mathrm{E}^{-7}$ & 0.67 \\
\hline $\begin{array}{c}\text { PERE } \\
\text { MJ }\end{array}$ & 260 & 1.20 & 0.79 & 0 & 0.01 & 240 & 310 & 16 & 0.11 & 820 \\
\hline $\begin{array}{c}\mathbf{F W} \\
\mathrm{m}^{3}\end{array}$ & 11 & 0.10 & 0.07 & 0 & $8.2 \mathrm{E}^{-3}$ & 1.60 & 25 & 75 & $3.8 \mathrm{E}^{-4}$ & 110 \\
\hline $\begin{array}{c}\text { CRU } \\
\mathrm{kg}\end{array}$ & 0 & 0 & 13 & 0 & 0 & 0.13 & 0 & 0 & 120 & 130 \\
\hline $\begin{array}{c}\text { MFR } \\
\mathrm{kg}\end{array}$ & 0 & $5.5 \mathrm{E}^{-3}$ & 290 & 0 & 0 & 24 & 0 & 0 & 3800 & 4100 \\
\hline
\end{tabular}

The largest contribution to AP and EP has aerated concrete blocks (11-15\%), concrete structures $(34-14 \%)$, waterproofing $(7-15,7 \%)$, plasterboard tiles $(4 \%)$ and aluminium windows (6-11\%). Reinforced concrete structures (15-37.6\%), impregnated wood materials $(47.4 \%)$ and aerated concrete blocks $(9.61-11.7 \%)$ significantly contribute to the POCP creation. These materials also have the highest percentage of ODP production. Sheep wool and cellulose insulation, ceramic tiles and plumbing products have the greatest impact on the ADPE impact.

\section{Conclusion}

This article shows that the correct selection of building materials as well as technologies related to building operation has significant impact reducing negative environmental impacts. The results of the comparison showed that FH 2 is the most sustainable building. External walls are made by load-bearing wooden frame system filled with straw insulation, which is clad with wood and subsequently plastered with clay plaster. It is roofed with a vegetation roof on a bearing wooden rafter system, insulated with blown wood fibre. A gas boiler was chosen as the heating source. GWP is a key indicator, given the high risk of greenhouse gas emissions, the concentration of which in the atmosphere causes global climate change. The phase of operating energy (B6) at this global potential has the greatest environmental impacts from the entire life cycle of buildings. 
The benefits of green buildings include lower operating costs, increased health and productivity, and positive environmental impacts.

This paper was financially supported by the Slovak Grants No. 1/0512/20 and 1/0648/17.

\section{References}

[1] Zhang, X. B., Bu, Z. C., Peng, F. P., \& Zhang, Y., Nuclear Physics A, (2015).

[2] Zuo, J., \& Zhao, Z. Y., Renewable and sustainable energy reviews, 30, 271-281, (2014).

[3] Tam, V. W., \& Tam, C. M., Building Research \& Information, 36(1), 37-43. (2008).

[4] International Energy Agency, Transition to Sustainable Buildings: Strategies and Opportunities to 2050, https://www.iea.org/etp/buildings/ , (2013).

[5] Cole, R. J., Building Research \& Information, 28(5-6), 304-309, (2000).

[6] World Green Building Council, The Business Case for Green Building - a Review of the Costs and Benefits for Developers, Investors and Occupants, https://www.worldgbc.org/news-media/business-case-green-building-review-costsand-benefits-developers-investors-and-occupants, (2013).

[7] Zhu, Y., \& Lin, B., Energy and Buildings, 36(12), 1287-1297, (2004).

[8] Fuerst, F., \& McAllister, P., Energy Policy, 39(10), 6608-6614, (2011).

[9] Zhou, Y., State power and environmental initiatives in China: Analyzing China's green building program through an ecological modernization perspective, Geoforum, 61, 1-12. (2015).

[10] Kok, N., McGraw, M., \& Quigley, J. M., American Economic Review, 101(3), 7782, (2011).

[11] Zhang, L., Wu, J., \& Liu, H., Journal of cleaner production, 172, 2234-2245, (2018).

[12] eToolLCD, https://etoollcd.com, (2019). 